\title{
Effect of transcutaneous electrical nerve stimulation with stretching and exercises in restless leg syndrome among pregnant women
}

\begin{abstract}
Background: Restless legs syndrome also known as Willis-Ekbom disease is a sensor motor disorder composed of an urge to move with or without associated discomfort that occurs with inactivity and improves with movement. The symptoms occur in leg muscles like calves and thighs. The feet and arms also affected and it causes the functional disability in pregnant women. Sleep disorder is typically the biggest complication of this condition. Unpleasant sensations are deep in the legs. Sensations appear during periods of rest or inactivity particularly in the evening and at night and relived by movement. Prevalence of RLS among pregnant women ranged from 10 to $34 \%$.
\end{abstract}

Aim: The aim of the study is to prove the effect of Transcutaneous Electrical Nerve
Stimulation (TENS) with Stretching and Exercises in restless leg syndrome among pregnant women.

Objectives: To determine and prove the effectiveness of TENS with stretching to relive pain and improve the functional ability in restless leg syndrome among pregnant women.

Materials and Methods: A Quasi-experimental study was conducted on 30 subjects using convenient sampling technique based on inclusion and exclusion criteria. Subjects were allotted into 2 groups i.e., Group A with 15 subjects and Group B with 15 subjects using odd even method of sampling. Experimental group will be treated with TENS and Stretching. Control group will be treated with exercises and massage.

Result: Statistical Analysis of Post test for pain and functional performance revealed that patients who received Transcutaneous Electrical Nerve Stimulation (TENS) with Stretching in Group A showed marked improvement compared to patients who received Massage and Exercises in Group B.

Conclusion: From the result of the study it concluded that Transcutaneous Electrical Nerve Stimulation (TENS) with Stretching is more effective than massage and exercises in subjects with restless leg syndrome among pregnant women.

Keywords: restless leg syndrome, sensor motor neurological disorder, transcutaneous electrical nerve stimulation, stretching, exercises, pregnant women, pain, functional ability, massage
Volume II Issue 2 - 2020

\author{
S Jenifer Augustina,' MG Kaviya, ${ }^{2}$ D Indrani' \\ 'Master of Physiotherapy (Obstetrics and Gynecology), Saveetha \\ College of Physiotherapy, Saveetha Institute of Medical and \\ Technical Sciences, India \\ 2Bachelor of Physiotherapy Saveetha College of Physiotherapy, \\ Saveetha Institute of Medical and Technical Sciences, India
}

\begin{abstract}
Correspondence: $\mathrm{S}$ Jenifer Augustina, PhD, Tutor, Master of Physiotherapy (Obstetrics and Gynecology), Saveetha College of Physiotherapy, Saveetha Institute of Medical and Technical Sciences, Prince apartment, near tollgate, Picasso C block 304 Sriperumbudur, Tamilnadu, Chennai, India, Tel 9962448947, Email Jenifer.augustin@gmail.com
\end{abstract}

Received: January 30, 2020 | Published: March 18, 2020
Abbreviations: TENS, transcutaneous electrical nerve stimulation; NPRS, numeric pain rating scale; RLS, restless leg syndrome; RLSQ, restless leg syndrome questionnaire

\section{Introduction}

During pregnancy, women undergo significant anatomical and physiological changes for developing fetus and prepare mother for labor and delivery. The changes in body size and shape are part of problem for pregnant women and for some women the complaints are specific. Sleep complaints are quit common during pregnancy ${ }^{1}$ and this is consistent with diagnosis of Restless legs syndrome also known as Willis-Ekbom disease ${ }^{2}$ is an sensor motor disorder ${ }^{3,4}$ composed of $^{2}$ an urge to move with or without associated discomfort that occurs with inactivity and improves with movement. The symptoms occur in leg muscles like calves and thighs. ${ }^{5}$ The feet and arms also affected and it causes the functional disability in pregnant women. Sleep disorder ${ }^{6}$ is typically the biggest complication of this condition. Unpleasant sensations ${ }^{7}$ are deep in the legs. Sensations appear during periods of rest or inactivity particularly in the evening and at night and relived by movement. Prevalence of $\mathrm{RLS}^{8,9}$ among pregnant women ranged from 10 to $34 \%$. Stress leg syndrome or otherwise called as Willis-ekbom disease. The potential pharmacological treatment of RLS during pregnancy is dopaminergic agents ${ }^{10}$ like ropinirole, pramipexole, benzodiazepine, Anti-epileptic drugs like gabapentin and iron supplement is recommended when pregnant women is diagnosed as iron-deficient. However, these medications during pregnancy has some effects on fetus like congenital malformations and there is a lack of good evidence for recommendations of these drugs to pregnant women for treating RLS and current practice focuses on counseling and iron therapy. Sleep hygiene should be correct before all pharmacological treatments. Most of the studies showed that lifestyle changes and massage during pregnancy improved prognosis in RLS. So in our study, we would like to compare this conventional treatment with my experimental treatment i.e. stretching and AROM Exercises. Transcutaneous electrical current (TENS) is a low voltage 
electrical current which is used to treat pain and it has been used by pregnant women for many years without reported any side effects. Some articles suggested that it is safe to use of TENS in pregnant women present with musculoskeletal pain like low back pain, pelvic girdle pain. This study presents guidelines for the use of TENS in restless leg syndrome. The main aim of study is to find the effect of Transcutaneous Electrical Nerve Stimulation (TENS) with Stretching Exercises to relive pain and improve the functional ability in restless leg syndrome among pregnant women.

\section{Methods and materials}

A Quasi-experimental study was conducted on 30 subjects using convenient sampling technique based on inclusion and exclusion criteria. Pregnant women between 25-30years of age, urge to move the legs and unpleasant sensation in the legs and III Trimester pregnant women are included in the study. Pregnant women with Periodic limb movement disorder, Leg Cramps, Leg injuries, Myalgia, Peripheral Neuropathy, Anxiety, Leg oedema, Insomnia, Iron deficiency and any other Discomforts are excluded from the study. Subjects were allotted into 2 groups i.e., Group A with 15 subjects and Group B with 15 subjects using odd even method of sampling. After receiving informed consent from the subjects they were explained about safety and simplicity of the procedure. Detailed explanation is given about Restless Leg Syndrome Questionnaire (Rating Scale) and Numeric Pain Rating Scale (NPRS) which is having their own reliability and validity. They were asked to rate their level of symptoms in restless leg syndrome Questionnaire and rate their level of pain using Numeric pain rate scale and the pre test values are documented. Experimental group were treated with TENS and Stretching. Control group were treated with exercises and massage. After 1week of intervention post test values are documented from both A and B Group.

\section{Experimental group A: Stretching}

Calf stretch: Stretch out your arms is flat against a wall so that your palms and your elbows are nearly straight. Slightly bend your right knee and step your left leg back a foot (or) two positioning its heel and foot flat on the floor. Hold for 20 to 30 seconds. Next bend your left knee while keeping your heel and foot flat on the floor, for a deeper stretch move foot back a bit. Switch legs and repeat it for 10 times.

Front thigh stretch: Standing parallel to a wall for balance pull one of your ankles toward your rear while keeping the other leg straight .Hold for 20 to 30 seconds. Switch legs and repeat it for 10 times.

Hip flexor stretch: Place the back of a chair against the wall for support and stand facing the chair. Raise your left foot up and rest it flat on the chair with your knee bent (or) try placing your foot on a stair while holding the railing for balance. Keeping your spine as neutral as possible, press your pelvis forward gently until you feel a stretch at the top of your right thigh. Rour pelvis will move forward only a little. Hold for 20 to 30 seconds. Switch legs and repeat it for 10 times.

Transcutaneous electrical nerve stimulation (tens): The patients are positioned comfortably in chair, provided with back rest and asked to keep the part treated to be relaxed and instructed to intimate if any discomfort or pain is felt. The electrodes are placed on the calf region bilaterally and these electrodes deliver electrical impulses to nerves and blocks pain signals to brain. Low frequency current with pulsed mode of $1-5 \mathrm{HZ}$ are used with duration of 10 minutes with $10 \mathrm{Ma}$ intensity.

\section{Control group B:Active range of motion exercises}
a. Dorsiflexion
b. Plantar flexion
c. Knee flexion
d. Knee extension

Massage: Massage leg before going to bed. Leg massages can help relax your muscles and encourage blood circulation. Using gentle kneading motions to massage your legs every night before bed. Massage is frequently recommended as a way of getting relief from restless leg syndrome. Increasing circulation in the hamstrings can relieve uncomfortable sensations in the legs.

Outcome measures:

- Restless Leg Syndrome Questionnaire (Rating Scale)

- Numeric Pain Rating Scale (NPRS)

\section{Restless leg syndrome questionnaire (rating scale)}

It is a rating scale for the Restless leg syndrome. Ask the patient to rate her symptoms for the following 10 questions. Very Severe $=31-40$ points, Severe $=21-30$ points, Moderate $=11-20$ points, Mild $=1-10$ points, None $=0$ points using these points the severity are measured.

1. Overall, how would you rate the RLS discomfort in your legs or arms?
(4) Very severe
(3) Severe
(2) Moderate
(1) Mild
(0) None

2. Overall, how would you rate the need to move around because of your RLS symptoms?
(4) Very severe
(3) Severe
(2) Moderate
(1) Mild
(0) None

3. Overall, how much relief of your RLS (arm or leg discomfort) do you get from moving around?
(4) No relief
(3) Slight relief
(2) Moderate relief
(1) Either complete or almost complete relief
(0) No RLS symptoms and therefore question does not apply

4. Overall, how severe is your sleep disturbance from your RLS symptoms?

(4) Very severe 
(3) Severe

(2) Moderate

(1) Mild

(0) None

5. How severe is your tiredness or sleepiness from your RLS symptoms?
(4) Very severe
(3) Severe
(2) Moderate
(1) Mild
(0) None

6. Overall, how severe is your RLS as a whole?
(4) Very severe
(3) Severe
(2) Moderate
(1) Mild
(0) None

7. How often do you get RLS symptoms?

(4) Very severe (This means 6 to 7 days a week.)

(3) Severe (This means 4 to 5 days a week.)

(2) Moderate (This means 2 to 3 days a week.)

(1) Mild (This means 1 day a week or less.)

(0) None

8. When you have RLS symptoms, how severe are they on an average day?

(4) Very severe (This means 8 hours per 24 hour day or more.)

(3) Severe (This means 3 to 8 hours per 24 hour day.)

(2) Moderate (This means 1 to 3 hours per 24 hour day.)

(1) Mild (This means less than 1 hour per 24 hour day.)

(0) None

9. How severe is the impact of your RLS symptoms on your ability to carry out a satisfactory family, home, social, school, or work life/ overall daily affairs?
(4) Very severe
(3) Severe
(2) Moderate
(1) Mild
(0) None

10. How severe are your RLS symptoms on mood disturbance (angry, depressed, sad, anxious, or irritable)?
(3) Severe
(2) Moderate
(1) Mild
(0) None

\section{umeric pain rating scale (NPRS)}

The Numeric Pain Rating Scale (NPRS) is a unidimensional measure of pain intensity in adults, including those with chronic pain the 11-points numeric scale ranges from "0" representing one pain extreme to "10" representing the other pain extreme. The NPRS is easy to administer and score (Figure 1) (Figure 2) (Table 1) (Table 2).

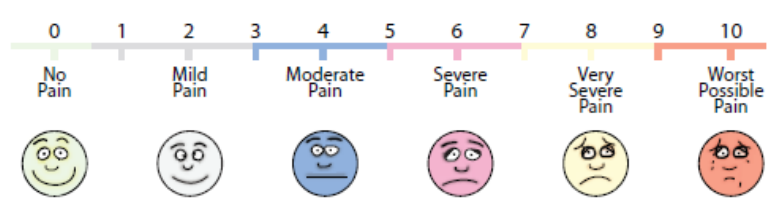

Figure I Comparison of pre-test and post-test values of NPRS and RLS in group $A$.

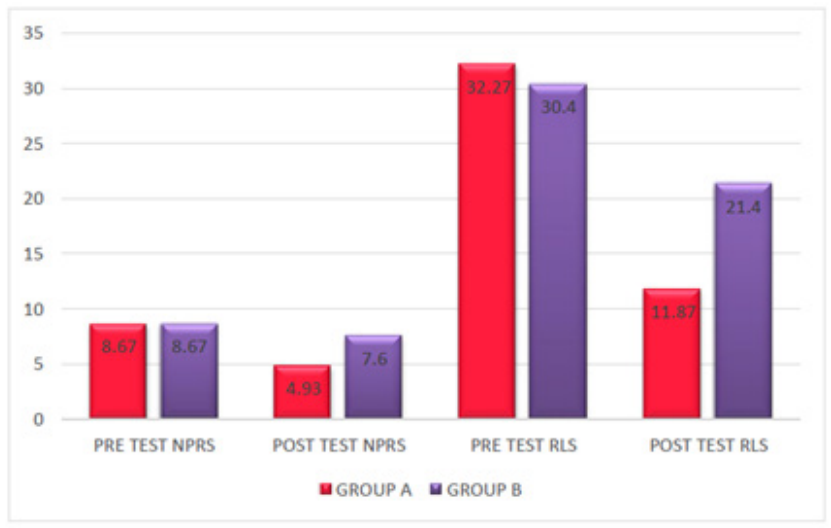

Comparison of pre-test and post test values of NPRS AND RLS QUESTIONNAIRE in group $A$ and group $B$

Figure 2 Comparison of pre-test and post test values of NPRS and RLS questionnaire in group $\mathrm{A}$ and group $\mathrm{B}$.

Table I Comparison of pre-test and post-test values of NPRS and RLS in group $\mathrm{A}$

\begin{tabular}{|c|c|c|c|c|c|}
\hline \multicolumn{2}{|c|}{ Experimental group IL-P A) } & \multirow{2}{*}{$\begin{array}{l}\text { Mean } \\
8.67\end{array}$} & \multirow{2}{*}{$\begin{array}{l}\text { Standard } \\
\text { deviation } \\
\text { (sd) }\end{array}$} & \multirow[t]{2}{*}{$\begin{array}{l}\text { t } \\
\text { value }\end{array}$} & \multirow[t]{2}{*}{$\begin{array}{l}P \\
\text { value }\end{array}$} \\
\hline & Pre-Test & & & & \\
\hline \multirow[t]{3}{*}{ NPRS } & & & & 0 & $<0.0001$ \\
\hline & Post-Test & 4.93 & 0.96 & & \\
\hline & Pre-Test & 32.27 & 4.1 & & \\
\hline \multirow{2}{*}{$\begin{array}{l}\text { RLS } \\
\text { QUESTIONNAIRE }\end{array}$} & & & & 1.4246 & $<0.0001$ \\
\hline & Post-Test & 11.87 & 1.55 & & \\
\hline
\end{tabular}

(4) Very severe 
Table 2 comparison of pre-test and post-test values of NPRS and RLS QUESTIONNARIE in group B

\begin{tabular}{|c|c|c|c|c|c|}
\hline \multicolumn{2}{|c|}{ Conventional group (group B) } & \multirow{2}{*}{$\begin{array}{l}\text { Mean } \\
8.67\end{array}$} & \multirow{3}{*}{$\begin{array}{l}\begin{array}{l}\text { Standard } \\
\text { deviation } \\
\text { (SD) }\end{array} \\
0.72 \\
0.63\end{array}$} & \multirow{3}{*}{$\begin{array}{l}\mathbf{t} \\
\text { value } \\
0\end{array}$} & \multirow{3}{*}{$\begin{array}{l}\mathbf{p} \\
\text { value }\end{array}$} \\
\hline & Pre-test & & & & \\
\hline NPRS & & & & & \\
\hline \multirow{2}{*}{$\begin{array}{l}\text { RLS } \\
\text { QUESTIONNAIRE, }\end{array}$} & Pre-test & 30.4 & 3 & \multirow{2}{*}{12.6466} & \multirow{2}{*}{$<0.0001$} \\
\hline & Post-test & 21.4 & 2.47 & & \\
\hline
\end{tabular}

a. On a scale of 0 to 10 , with 0 being no pain at all and 10 being the worst pain imaginable, how you would rate your pain RIGHT NOW. 012345678910 No Worst Pain Imaginable

b. On the same scale, how would you rate your USUAL level of pain during the last week. 012345678910

c. No Worst Pain Imaginable

\section{Results}

From statistical analysis made with the quantitative data revealed statistically significant difference between the Group A and Group $B$ and also within the group .The post-test mean value of NPRS in group A is 4.93 and post-test mean value of NPRS in group B is 7.60. This shows that Numeric Pain Rating Scale Scores in Group A were Comparatively lesser than Group B,P $<0.0001$.

The post- test mean value of RLS Questionnaire in group A is 11.87 and post-test mean value of RLS Questionnaire of group B is 21.40.This shows that Restless Leg Syndrome Questionnaire Rating Scale scores in Group A were Comparatively lesser than Group B, $\mathrm{P}<0.0001$.

Statistical Analysis of Post test for pain and functional performance revealed that there was high statically significant difference seen between Group A and Group B.

\section{Discussion}

Restless leg syndrome (RLS) is a common sleep related sensorimotor neurological disorder commonly associated with pregnancy. It is characterized by an urge to move the limbs due to unpleasant sensation and it is associated with periodic limb movements. The various unpleasant sensations are "creeping", "crawling", "tingling", "tickling" and "numbness". This abnormal sensation felt deeply in the bones not superficially. The prevalence of restless leg syndrome during pregnancy is around three times greater than the general population. The reported prevalence of RLS during pregnancy is about 10 to $34 \%$. The symptoms of RLS first developed during pregnancy and the prevalence was found to be increase in advancing gestational age and the prevalence gets severed during the third trimester and ends in delivery. The symptoms occur at rest and relieved by active movements and it usually worsens in the evening. More than $80 \%$ of RLS patients experienced periodic leg movements during their sleep and it is characterized by involuntary muscular jerks in the lower limb. This condition also causes leg pain and cramps. The sleep disruption is the major complaint of RLS patients and it impact on health and daytime functioning. The pathophysiology of Restless leg syndrome is mainly focusing on genetic predisposition, dopaminergic dysfunction and abnormal iron metabolism. It is considered to be a central nervous system related disorder with no specific lesion has been found. There are some evidences showing raising estrogen levels may contribute RLS. The complications of RLS are day time sleepiness, low energy, irritability and depression. In group A post intervention mean of Numeric Pain Rating Scale (NPRS) was 4.93 and in group B post intervention mean of NPRS was 7.60. In group A post intervention mean of Restless Leg Syndrome Questionnaire (Rating Scale) was 11.87 and in group B post intervention mean of RLSQ (Rating Scale) was 21.40.which shows statistical significant difference within the group

The present study reveals that patients who received Transcutaneous Electrical Nerve Stimulation (TENS) with Stretching in Group A showed marked improvement. Compared to patients who received Massage and Exercises in Group B.

\section{Conclusion}

The study concluded that both the groups A and B resulted in positive outcomes, but group A with Stretching and Transcutaneous Electrical Nerve Stimulation (TENS) showed a higher level of positive outcome in terms of decreasing pain and improving functional ability. when compared to group B with Massage and Exercises in subjects with Restless Leg Syndrome among Pregnant women.

\section{Limitations of the study}

Sample size of the study is small, Long term follow up was not done, Shorter duration of the treatment are the limitations.

\section{Acknowledgements}

None.

\section{Funding}

None.

\section{Conflicts of interest}

The authors declare there are no conflicts of interest.

\section{References}

1. Aarti G, Courtney CB, Carolyn MDA. Restless leg syndrome in pregnancy. Obstet Med. 2015;8(3):121-125.

2. Abdurrahman N, Gulcin BS, Rahime A, et al. A prospective study of willis-ekbom disease/restless legs syndrome during and after pregnancy. Sleep Med. 2015;16(9):1036-1040.

3. Shiyi G, Jinsha H, Haiyang J, et alg. Restless legs syndrome: from pathophysiology to clinical diagnosis and management. Front Aging Neurosci. 2017;9:171.

4. Prachaya S, Sanjay P, Mark H. Restless leg syndrome and pregnancy: a review. Parkinsonism Relat Disord. 2014;20(7):716-722.

5. Ekbom K, Ulfberg J. Restless leg syndrome. Journal of Internal Medicine.2009;266(5):419-431.

6. Galit LD, Lynda DL, Kerby S, et al. Restless legs syndrome and sleepwake disturbances in pregnancy. J Clin Sleep Med. 2017;13(7):863-870.

7. Paul EC, Shaun TOK. Restless leg syndrome: is it a real problem? Ther Clin Risk Manag. 2006;2(4):465-475.

8. Maurice MO, Ruth $\mathrm{OH}$, Michael VV. Epidemiology of restless legs syndrome: A synthesis of the literature. Sleep Med Rev. 2012;16(4):283295. 
9. Gupta R, Dhyani M, Kendzerska T, et al. Restless legs syndrome and pregnancy: prevalence, possible pathophysiological mechanisms and treatment. Acta Neurol Scand. 2016;133(5):320-332.
10. Chaudhuri KR, Appiah K, Trenkwalder. The restless leg syndrome. Journal of Neurology Neurosurgery \& Psychiatry. 2001;71:143-146. 